\title{
Atividade física e prevalência de quedas em idosos residentes no sul do Brasil
}

\section{Physical activity and prevalence of falls among elderly living in southern Brazil}

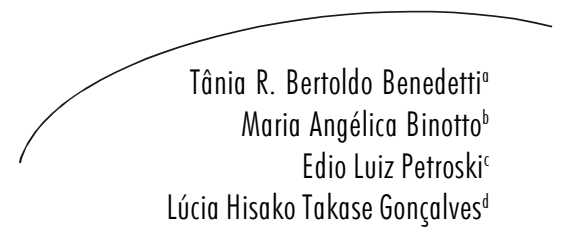

Resumo

O objetivo do estudo foi investigar a relação entre os níveis de atividade física e a prevalência de quedas em idosos residentes em Florianópolis, SC, Brasil. Trata-se de estudo com amostragem do tipo probabilística e estratificada por setor censitário e sexo, com 875 idosos. Os instrumentos adotados para a coleta de dados foram o Questionário Brazil Old Age Schedule (BOAS) e o Questionário Internacional de Atividades Físicas (IPAQ). Os resultados apontaram que 59,3\% dos idosos eram considerados mais ativos. Idosos com acometimento de quedas durante o peńodo de três meses que antecederam a entrevista foram $11,4 \%$, dos quais a maioria era mais ativa. Houve associação estatística entre quedas e as variáveis: problemas nos pés $(p=0,002)$ e articulações $(p=0,006)$. Sendo assim, conclui-se que a educação para os cuidados de prevenção de quedas se faz necessária, ao mesmo tempo em que se estimulem atividades físicas livres de riscos entre a população idosa.

\section{Abstract}

This study aimed to investigate the relation between the physical activities level and falls prevalence among elderly people in the city of Florianopolis, state of Santa Catatina, Brazil. It is an epidemiological , cross-sectional and descriptive study, carried out in the city of Florianópolis, Brazil. The sampling, of statistical type and stratified by census section and sex, resulted

Comespondência / Comespondenœ

Tânia R. Bertoldo Benedetti

Universidade Federal de Santa Catarina

Departamento de Educação Física, Centro de Despostos

Campus Universitário, Caixa Postal 476 - Trindade

88040-900 - Florianópolis, SC, Brasil

E-mail: benedetti@cds.ufsc.br

\section{Palavras-chave: \\ idoso; atividade \\ motora; acidentes \\ por quedas; \\ prevalência; \\ estudos transver- \\ sais; amostragem \\ estratificada; \\ probabilidade; \\ questionários; \\ Florianópolis, SC, \\ Brasil}


in a sample of 875 senior citizens. The instruments adopted for data collection were the Questionnaire Brazil Old Age Schedule (BOAS) and the International Questionnaire of Physical Activities (IPAQ) long version. Results pointed out that $59.3 \%$ of seniors were considered more active ones. Seniors who suffered falls during the period of three months before the interview reached the amount of $11.4 \%$ and most of them were more active people. There was statistical association between falls and the variables: problems in the feet $(p=0,002)$ and articulations $(p=0,006)$. It concludes that it is necessary to prevent falls and, at the same time, to stimulate physical activities free from risks for the elderly population.
Key words: age;

motor activity; accidental falls; prevalence; crosssectional studies; stratified sampling; probability; questionnaires; Florianópolis, SC, Brasil

\section{INTRODUÇÃO}

O aumento da expectativa de vida tem grande impacto sobre o envelhecimento da população. No Brasil, no início do século $\mathrm{XX}$, o tempo médio de vida era 33 anos e atualmente (2000) é de 71,3 anos. ${ }^{1} \mathrm{O}$ ideal da longevidade é que as pessoas envelheçam com boa saúde, alto grau de independência e autonomia. A queda tem sido uma das principais causas de perda da independência, com muitas conseqüências, inclusive morte prematura.

A queda é definida como um deslocamento não-intencional do corpo para um nível inferior à posição inicial, com incapacidade de correção em tempo hábil, determinado por circunstâncias multifatoriais que comprometem a estabilidade. ${ }^{2}$

Em pessoas idosas, a queda é uma causa crescente de lesões, gastos com tratamento e mortalidade. Além disso, produz restrição das atividades da vida diánia, incapacidades, declínio na saúde e aumento do risco de institucionalização, propiciando não apenas prejuízo físico, mas também psico- lógico. Os custos econômicos que decorrem de quedas são altos: uma fratura de colo de fêmur custa em média 7.000 dólares; uma fratura de vértebra, 1.200. Contudo, os maiores problemas são as limitações que essas fraturas produzem nos idosos, pois cerca de 15\% deles passam a sofrer de incapacidades e requerem cuidado de saúde ${ }^{3}$ após as quedas.

Segundo a Organização Mundial da Saúde, ${ }^{4}$ a queda tem sido um dos problemas mais importantes e comuns relatados entre os idosos, aumentando progressivamente com o avanço da idade das pessoas, em ambos os sexos.

No âmbito mundial, a literatura tem registrado aumento na freqüência de queda de aproximadamente $40 \%$ ao ano em idosos com idade acima de 80 anos, e cerca de $30 \%$ em idosos com mais de 65 anos. ${ }^{5,6}$ As estimativas apontam que uma em cada três pessoas com idade igual ou supenior a 65 anos cai anualmente nos Estados Unidos. Na Pensilvânia, $62 \%$ de todos os traumas em pessoas dessa faixa etánia decorreram de quedas. ${ }^{7}$ No Brasil, a ocorrência de quedas por faixa etánia, a cada ano, atinge 32\% dos 
idosos entre 65 e 74 anos, 35\% de 75 a 84 anos e $51 \%$ acima de 85 anos. De modo geral, 30\% dos idosos brasileiros caem ao menos uma vez ao ano. ${ }^{2}$

Portanto, as quedas entre pessoas idosas constituem um dos principais problemas clínicos e de saúde pública, por sua alta incidência e pelas conseqüentes complicações para a saúde, além dos altos custos assistenciais. ${ }^{6,8,9}$

Programas de exercícios físicos que aumentam significativamente a força muscular, mantendo a composição e o peso corporal e melhorando o equilíbrio, podem diminuir quedas entre os idosos, tornando-se uma forma efetiva de prevenção. ${ }^{9,10}$ Além disso, 0 exercício físico proporciona aumento do contato social, diminui os niscos de doenças crônicas, melhora a saúde física e mental e a performance funcional, assegurando independência e autonomia por mais tempo. ${ }^{11}$

Embora sejam evidentes o aumento da incidência de quedas entre os idosos e os benefícios do exercício físico, poucos estudos relacionam as quedas com os níveis de atividades físicas. Sendo assim, o objetivo deste estudo foi investigar a relação entre os níveis deatividades físicas e a prevalência de quedas entre idosos residentes no sul do Brasil.

\section{METODOLOGIA}

Trata-se de estudo de abordagem epidemiológica do tipo transversal e descritivo, realizado na cidade de Flonianópolis, capi- tal do Estado de Santa Catanina, Brasil. Flonianópolis (2000) conta com um total de 28.816 pessoas idosas, correspondendo a $8,4 \%$ de sua população. ${ }^{12}$

A amostragem foi do tipo probabilístico, com técnica de seleção estratificada por setor censitánio e sexo, e investigou 875 idosos, sendo 437 homens e 438 mulheres. 0 intervalo de confiança adotado foi de $95 \%$, sendo a unidade amostral o domicilio. O projeto foi aprovado pelo Comitê de Ética em Pesquisa com Seres Humanos da UFSC (processo 051/2001).

Os instrumentos adotados para a coleta de dados foram: Questionánio Brazil Old Age Schedule (BOAS), que tem como objetivo assegurar informações sobre as pnincipais caracteństicas sociodemográficas, condições de vida, necessidades e problemasque afetam a população idosa e Questionánio Internacional de Atividades Físicas (IPAQ) versão longa, que tem como objetivo mensurar as atividades físicas realizadas nos domínios do trabalho, do transporte, das atividades domésticas e de lazer.

Para o presente estudo, foram analisados os dados das sessões do BOAS referentes às variáveis socioeconômicas e à saúde física (acometimento de quedas nos três meses que antecederam o estudo, uso de medicamentos, doenças), relacionados com os dos níveis de atividade física. Paraverificar o nível de atividade física, utilizou-se o IPAQ, versão longa. O IPAQ é um questionánio que mensura o tempo gasto em atividades físicas moderadas e/ ou vigorosas nos domínios do trabalho, transporte, atividades do- 
mésticas e de lazer durante uma semana usual. Foram considerados como menos ativos os idosos que, no somatónio das atividades físicas nos diferentes domínios, obtiveram menos de 150 minutos em atividades moderadas ou vigorosas por semana, e mais ativos os que obtiveram um escore igual ou superior a 150 minutos. $^{13}$

Para análise da condição econômica, osparticipantes foram divididos em tês categonas derendafamiliar: (1)até2salánosmínimos(2)de2 a6salániosmínimose(3)aàmade6salánosmínimos Entende-se que no primeiro parâmetro encontram-se as famílias no limite da pobreza. ${ }^{14}$

A condição de escolaridade foi categorizada em baixa (analfabetos); média (menos de 8 anos de escolaridade); e alta (mais de 8 anos de escolaridade). Tais parâmetros estão baseados nos estudos da Fundação Seade (1992). ${ }^{14}$

Os dados provenientes do questionánio BOAS e do IPAQ foram tratados pelo pro- grama SPSS 11.0, incluindo a análise descritiva, teste qui-quadrado e a análise de regressão logística (OddsRatio).

\section{RESULTADOS}

Os idosos de Flonianópolis tinham a média etána de 71,6 \pm 7,9 anos; 61,4\% eram casados, $66,6 \%$ viviam com os fillhos e $13,3 \%$ residiam so zinhos. Em relação à escolanidade, os idosos, em sua maionia, sabiam ler e escrever (80\%) e estudaram até a $4^{0}$ séne do ensino fundamental (42,7\%). Com curso supenior havia $11,9 \%$ dos idosos, a maionia do sexo masculino (18,8\%).

Para análise do nível de atividade física por meio do IPAQ, foram apresentados a freqüência e o percentual por sexo e nível de atividade física em cada domínio (Tabela 1). Para verificar o tempo gasto em atividades físicas moderadas e vigorosas, foram realizados as médias e os desvios-padrão nos diferentes domínios.

Tabela 1 - Percentual nos domínios da atividade física dos idosos de Florianópolis segundo sexo e classificados em níveis de atividade física, 2002.

\begin{tabular}{|c|c|c|c|c|c|c|c|c|}
\hline \multirow{3}{*}{$\begin{array}{l}\text { Domínios } \\
\text { Atividades } \\
\text { Físicas - } \\
\text { IPAQ }\end{array}$} & \multicolumn{6}{|c|}{ Nível de Atividade Física } & \multirow{2}{*}{\multicolumn{2}{|c|}{$\begin{array}{l}\text { Atividades } \\
\text { Físicas } \\
\text { Min/sem }\end{array}$}} \\
\hline & \multicolumn{3}{|c|}{ Menos ativos (PA1) } & \multicolumn{3}{|c|}{ Mais ativos (PA2) } & & \\
\hline & $\begin{array}{c}\text { Masculino } \\
\%\end{array}$ & $\begin{array}{c}\text { Feminino } \\
\%\end{array}$ & $\begin{array}{c}\text { Total } \\
\%\end{array}$ & $\begin{array}{c}\text { Masculino } \\
\%\end{array}$ & $\underset{\%}{\text { Feminino }}$ & $\begin{array}{c}\text { Total } \\
\%\end{array}$ & $\mathbf{X}$ & $\mathrm{dp}$ \\
\hline Total & 36,4 & 45 & 40,7 & 63,6 & 55,0 & 59,3 & 521,6 & 270,1 \\
\hline Trabalho & 90,4 & 96,6 & 93,5 & 9,6 & 3,4 & 6,5 & 48,2 & 240,4 \\
\hline Transporte & 76,4 & 84,5 & 80,5 & 23,6 & 15,5 & 19,5 & 157,1 & 194,1 \\
\hline Doméstica & 83,7 & 75,3 & 79,5 & 16,2 & 24,6 & 20,5 & 227,5 & 411,2 \\
\hline Lazer & 69,6 & 79 & 74,3 & 30,4 & 21 & 25,7 & 272,5 & 259,9 \\
\hline
\end{tabular}


No presente estudo, somando-se as atividades desenvolvidas nos diferentes domínios (trabalho, transporte, atividades domésticas e lazer), 59,3\% dos idosos foram considerados mais ativos. Os homens foram mais ativos do que as mulheres. A média de tempo despendido em atividades físicas moderadas e vigorosas foi de 521,6 minutos por semana. Analisando-se os domínios se- paradamente, constata-se que os homens são mais ativos no lazer (30,4\%), enquanto as mulheres são mais ativas nas atividades domésticas (24,6\%).

A condição de saúde dos idosos referentes a queda, problemas de saúde e medicamentos, considerando-se o nível de atividade física e o sexo, está apresentada na Tabela 2.

Tabela 2 - Condições de saúde dos idosos de Flonianópolis, segundo nível de atividade física e sexo, 2002.

\begin{tabular}{|c|c|c|c|c|c|c|}
\hline \multirow[b]{2}{*}{ Saúde } & \multicolumn{2}{|c|}{ Masculino } & \multicolumn{2}{|c|}{ Feminino } & \multicolumn{2}{|c|}{ Total } \\
\hline & $\begin{array}{c}\text { Menos } \\
\text { ativo (\%) }\end{array}$ & $\begin{array}{c}\text { Mais } \\
\text { ativo (\%) }\end{array}$ & $\begin{array}{c}\text { Menos } \\
\text { ativo (\%) }\end{array}$ & $\begin{array}{c}\text { Mais } \\
\text { ativo (\%) }\end{array}$ & $\begin{array}{c}\text { Menos } \\
\text { ativo (\%) }\end{array}$ & $\begin{array}{l}\text { Mais } \\
\text { ativo (\%) }\end{array}$ \\
\hline \multicolumn{7}{|c|}{ Ocorrência de queda } \\
\hline $\operatorname{Sim}$ & 4,8 & 8,4 & 3,4 & 6,2 & 4,1 & 7,3 \\
\hline Não & 31,6 & 55,2 & 41,6 & 48,8 & 36,6 & 52 \\
\hline \multicolumn{7}{|c|}{ Tem problema de saúde } \\
\hline $\operatorname{Sim}$ & 25,1 & 4,4 & 30,8 & 41,8 & 28 & 43,1 \\
\hline Não & 11,3 & 19,2 & 14,2 & 13,2 & 12,7 & 16,2 \\
\hline \multicolumn{7}{|c|}{ Ingestão de medicamentos } \\
\hline $\operatorname{Sim}$ & 27,5 & 45,1 & 31 & 42,5 & 29,2 & 43,8 \\
\hline Não & 8,9 & 18,5 & 13,9 & 12,6 & 11,4 & 15,6 \\
\hline \multicolumn{7}{|c|}{ Quantidade de medicamentos } \\
\hline Nenhum & 8,9 & 18,5 & 13,9 & 12,8 & 11,5 & 15,6 \\
\hline $1-3$ & 22,9 & 37,8 & 24 & 33,3 & 23,4 & 35,5 \\
\hline $4-6$ & 3,9 & 5,7 & 6,4 & 7,5 & 5,1 & 6,6 \\
\hline Mais de 7 & 0,7 & 1,6 & 0,7 & 1,4 & 0,7 & 1,5 \\
\hline
\end{tabular}

A ocomência de queda nos três meses que antecederam a pesquisa foi relatada por $11,4 \%$ dos idosos, dos quais a maiona era mais ativa. Dos idosos que caíram, 60\% conseguiram se levantar so zinhos do chão. Entre os que não conseguiram se levantar sozinhos, o tempo que o idoso permaneceu no chão após a queda vaniou de menos de um minuto (14\%) até mais de cinco minutos (13\%). Alguns necessitaram de ajuda de terceiros para levantar-se e outros apresentaram fraturas devido à queda.

Quanto ao nível de atividades físicas em ambos os sexos, os idosos considerados mais ativos caíram com mais frequiência. 
Em relação ao relato de problemas de saúde, tanto os mais ativos (43,8\%) quanto os menos ativos (28\%) mencionaram ser portadores de algum problema de saúde.

Quanto ao uso de medicamentos, 73\% dos idosos relataram estar usando medicação, 43,8\% dos quais foram consideradosmais ativos e 29,2\% menos ativos. Em relação à quantidade de medicamentos ingenidos pelos idosos, a maiona (59,9\%) utiliza 1 a 3 tipos de medicamentos diferentes dianiamente, não havendo diferença entre os sexos.
Ao analisar a ocorrência de quedas considerando-se as diferentes faixas etárias, observa-se que os idosos que mais sofreram quedas encontram-se na faixa etánia de 70 a 79 anos de idade, enquanto idosos que não sofreram quedas nos três meses que antecederam a pesquisa, no total de $47,2 \%$, encontram-se com idade inferior a 70 anos.

A associação de quedas com outras variáveis do estudo se apresenta na Tabela 3 .

Tabela 3 - Associação do acometimento de queda com variáveis socioeconômicas, atividade física e saúde em idosos de Flonianópolis.

\begin{tabular}{lcc}
\hline \multicolumn{1}{c}{ Variáveis } & Qui-quadrado $\left({ }^{2}\right)$ & $\mathrm{p}$ \\
\hline Faixa etária & 3,502 & 0,061 \\
Gênero & 1,040 & 0,332 \\
Escolaridade & 0,701 & 0,954 \\
Renda familiar & 6,270 & 0,177 \\
Atividade física & 1,040 & 0,308 \\
Problemas de Saúde & 2,754 & 0,064 \\
Problemas nos pés & 9,629 & $0,002^{*}$ \\
Problemas articulares & 7,860 & $0,006^{*}$ \\
Uso de bengala & 0,166 & 1,000 \\
Uso de medicamentos & 2,140 & 0,093 \\
\hline
\end{tabular}

*Significativo para $\mathrm{p}<0,05$

Em relação às vanáveis do estudo e sua associação com a queda, pode-se constatar que as variáveis problemas nos pés e problemas articulares ( $p=0,002$ e 0,006, respectivamente) estão associadas à ocorrência de queda nessa população.

Quando se utilizou a análise de regressão (oddsratio) com a ocomência de quedas com o gênero ( $\mathrm{OR}=0,088$ IC 0,45 - 1,05 $\mathrm{p}=0,08$ ), com o uso de medicamentos (OR $=1,444$ IC $0,871-2,396 p=0,155)$ e com 0 nível de atividade física (OR $=1,250$ IC 0,811 - 1,927 p=0,312), pôde-se observar que a razão de chance foi baixa com todas as variáveis. 


\section{DISCUSSÃO}

Os resultados encontrados mostram que a maionia dos idosos (59,3\%) da população estudada foi considerada mais ativa. Quando se analisou especificamente o domínio lazer, verificou-se que 30,4\% dos homens e 21\% das mulheres são considerados maisativos. Esse resultado é semelhante ao encontrado no estudo realizado por Yusuf etal., ${ }^{15}$ com idosos amenicanos, que apontou prevalência de 37\% dos homens e 24\% das mulheres mais ativos no lazer. $\mathrm{O}$ presente estudo evidenciou que os homens praticam mais atividades físicas no lazer do que as mulheres. O predomínio masculino em atividades físicas de lazeré observado em todas as capitais de estados brasileiros, tanto entre adultos como entre idosos. ${ }^{16}$ Contudo, a inatividade física observada em todos os domínios (trabalho, transporte, doméstica e lazer) foi duasvezes mais freqüente em homens (39,8\%) do que em mulheres (20,1\%). E entre pessoas com 65 anos ou mais, $64,5 \%$ dos homens e $50,3 \%$ das mulheres são inativos. ${ }^{16}$

A prática de atividade física sistemática contribui para manter e/ ou melhorar a força, a flexibilidade, a coordenação e o equilíbrio, elementos da aptidão física essenciais para manter a capacidade funcional do idoso por mais tempo. ${ }^{17,9}$ Além disso, a atividade física tem sido comprovada como fator de melhora da saúde global dos idosos, daí o seu incentivo ser importante medida de prevenção das quedas, por lhes proporcionar maior segurança na realização de suas atividades de vida diánia. ${ }^{18}$
A ocorrência de quedas nos três meses que antecederam este estudo foi relatada por $11,4 \%$ dos idosos. Tal prevalência foi infenor à encontrada no estudo realizado por Hawk etal., ${ }^{19}$ no qual 13\% dos idosos haviam sofido queda no mês anterior à pesquisa, dos quais $72 \%$ praticavam exercícios físicos regularmente, $26 \%$ os praticavam ocasionalmente e $2 \%$ não os praticavam.

Quando nos reportamos à ocorrência de quedas em idosos praticantes de atividades físicas, no peńodo de um ano que antecedeu a pesquisa, encontramos prevalências semelhantes entre os estudos: $44 \%{ }^{19}$ e $43 \%{ }^{20}$ dos idosos.

Em relação à ocorrência de quedas e 0 nível de atividade física, tanto os idosos menos ativos quanto os mais ativos as sofreram, embora entre os que caíram predominassem os mais ativos. Com isso, pode-se infenirque os idosos mais ativos andam mais e participam de diferentes atividades, expondo-se mais ao nisco de queda. Já os idosos menos ativos, com medo de cair e ficar com algum comprometimento físico, restringem ainda mais as reduzidas atividades que desempenham.

Segundo Tinetti ${ }^{21} \&$ Santariano etal., ${ }^{22} \mathrm{a}$ queda desenvolve nos idosos o medo de cair, levando-os a perder a autoconfiança para desenvolver atividades diánias e a optar por um estilo de vida inativo, que resulta em atrofia muscular e conseqüente diminuição da força muscular, além do aumento da dependência. Os resultados encontrados por Koepsell etal. ${ }^{23}$ apontam que somente $10 \%$ 
das quedas ocorreram durante a realização de atividades físicas moderadas e menos de $2 \%$ durante as atividades físicas vigorosas: as quedas ocorrem principalmente nas atividades diánias.

Na presente investigação, os homens tiveram ocorrência de queda maior do que as mulheres, ao contránio do que aponta a literatura, segundo a qual maiona das quedas ocorre entre idosos do sexo feminino, tendo como possíveis causas a maior prevalência de doenças crônicas e mais atividades domésticas. ${ }^{24}$

O uso de medicamentos foi relatado pela maionia dos idosos. Para Nnodim e Alexander, ${ }^{10}$ os medicamentos devem ser limitados àqueles absolutamente essenciais, em função do aumento do risco de quedas com o uso de vários tipos de medicamentos. Neste estudo, 11,7\% utilizam 4 a 6 tipos de medicamentos e 2,3\% usam de 7 ou mais medicamentos, embora não haja associação estatística com a ocorrência de quedas. Para Rao, ${ }^{25}$ o uso de quatro ou mais medicamentos foi associado fortemente com um risco aumentado de quedas. Estudo de coorte transversal realizado por Lawlor et al. ${ }^{26}$ sobre a ocorrência de quedas em 4.050 mulheres com idades entre 60-79 anos, nos 12 meses que antecederam a pesquisa, concluiu que as doenças crônicas e a utilização múltipla de medicamentos são importantes preditores de quedas.

Quanto à associação de quedas com as variáveis de informações gerais e de saúde
- faixa etánia, gênero, escolaridade, renda familiar, atividade física, problemas de saúde nos pés e nas articulações, uso da bengala e de medicamentos -, verificou-se associação de queda somente com problemas nos pés e nas articulações, embora Guimarães e Faninattii ${ }^{20}$ tenham encontrado associação da queda com o uso de medicamentos e a faixa etánia.

Os dados deste estudo apontam que os idosos mais acometidos por quedas se encontram na faixa etána de 70 a 79 anos de idade. No entanto, idosos acima de 80 anos tendem a sofrer mais quedas do que idosos de faixas etánias inferiores, apresentando nisco relativo de 1.7 de ocorrência de quedas. ${ }^{17,27}$

Ao que parece, as quedas sofridas por idosos de Flonianópolis se prendem a múltiplos fatores, não evidenciados estatisticamente neste estudo.

\section{CONCLUSÃO}

Os resultados do estudo demonstram que os idosos considerados fisicamente mais ativos tiveram maior incidência de queda do que os menos ativos. Quando considerado o sexo, observou-se que as quedas ocorreram mais entre os homens do que entre as mulheres. Sendo assim, conclui-se que a educação para os cuidados de prevenção de quedas se faz necessánia, ao mesmo tempo em que se estimulem atividades físicas livres de riscos à população idosa. 


\section{NOTAS}

a Professora Doutora, Departamento de Educação Física. E-mail: benedetti@cds.ufsc.br

b Mestre em Educação Física, Departamento de Educação Física E-mail: manbinotto@yahoo.com.br

c Professor Doutor, Departamento de Educação Física E-mail: petroski@cds.ufsc.br

d Professora Doutora, Departamento de Enfermagem. E-mail: lucia@nfr.ufsc.br

Parte da Tese: "Atividade Física: uma perspectiva de promoção da saúde do idoso no município de Florianópolis", defendida em dezembro de 2004, no Programa de Doutorado em Enfermagem, CCS/UFSC.

Financiado pelo Ministério da Saúde (Processo 4345/ 01); Conselho Nacional de Desenvolvimento Científi-

\section{REFERÊNCIAS}

1. IBGE. Síntese de indicadores sociais, 2004. Rio de Janeiro: IBGE. (2005). [acesso 2006 ago. 20]. Disponível em: URL: http:// www.ibge.gov.br/home/estatistica/ populacao/condicaodevida/indicadore

2. Pereira SRM, Buksman S, Perracini M, Py L, Barreto KML, Leite VMM Quedas em Idosos. Sociedade Brasileira de Geriatria e Gerontologia; 2001.

3. Carvalhaes N, Rossi E, Paschoal S, Perracini N, Perracini M, Rodrigues R. Quedas. Consenso de Gerontologia. Anais do $1^{0}$ Congresso de Genatria e Gerontologia; 1998; São Paulo. São Paulo; 1998. p. 5-18.

4. Organização Mundial da Saúde. Active ageing. A policy framework. A contribution of the World Health Organization to the Second United Nations World Assembly on Ageing. Madrid; 2002.

5. Powell LE, Myers, AM The Activitiesspecific Balance Confidence (ABC) scale. Journal Gerontol. A: Biol. Sci. Med. Sci 1995; 54: 165-71. co e Tecnológico (processo no . 520824/1997-0, bolsa de Iniciação científica); Coordenação de Aperfeiçoamento de Pessoal de Nível Superior (bolsa de doutorado sanduíche); e apoio técnico do Instituto Brasileiro de Geografia e Estatística (IBGE - seção de Florianópolis

\section{Agradecimentos}

Ao Ministério da Saúde (processo ${ }^{\circ}$ 4345/01); CNPq (processo no 520824/19970); CAPES (bolsa de doutorado sanduíche); Instituto Brasileiro de Geografia e Estatística (IBGE - seção de Flonianópolis).

\section{Tinetti ME Falls in Elderly Persons. N Engl J Med 2003; 348: 42-9.}

7. Marshall SW, Runyan CW, Yang J, CoyneBeasley T, Waller AE, Johnson R, Perkis D. Prevalence of Selected Risk and Protective Factors for Falls in the Home. Am J Prev Med 2005; 28 (1): 95-101.

8. Lajoie Y, Gallagher SP. Predicting falls within the elderly community: comparison of postural sway, reaction time, the Berg balance scale and the Activities-specific Balance Confidence scale for comparing fallers and nonfallers. Arch Gerontol Genatr 2004; 38(1): 11-25.

9. Spirduso WW. Dimensões físicas do envelhecimento. Barueni, SP: Manole; 2005.

10. Nnodim JO, Alexander NB. Assessing falls in older adults: a comprehensive fall evaluation to reduce fall risk in older adults. Geriatrics 2005; 60(10): 24-8.

11. Chanler J, Studensk S. Exercícios. In: Duthie EH, Katz PR. Geriatria prática. 
3.ed. Rio de Janeiro: Revinter; 2002. p.125-39.

12. IBGE. Senso demográfico 2000. Sinopse preliminar do censo demográico de 2000. Rio de Janeiro: IBGE; 2001. v. 7.

13. Pate RR, Pratt M, Blair SN, Haskell WL, Macera CA, Bouchard C, et al. Physical activity and public health: a recommendation from the Centers for Disease Control and Prevention and the American College of Sports Medicine. JAMA 1995; 273 (5): 402-07.

14. Fundação Seade. Fundação Sistema Estadual de Análise de Dados Pobreza e Riqueza: Pesquisa de Condições de Vida na Região Metropolitana de São Paulo. Educação e Renda. São Paulo: Fundação Seade;1992.

15. Yusuf, HR, et al. Leisure-time physical activity among older adults. Arch Intem Med 1996; 156: 1321-26.

16. Brasil. Ministério da Saúde. Vigitel Brasil 2006. Vigilância de fatores de risco e proteção para doenças crônicas por inquénito telefônico [CD]. Brasilia, DF; 2007.

17. Barnett A, Smith B, Lord SR, Williams M, Baumand A. Community-based group exercise improves balance and reduces falls in at-nisk older people: a randomized controlled trial. Age Ageing 2003; 32 (4): 407-14.

18. Barbosa MT. Como avaliar quedas em idosos? Revista Associação Médica Brasileira 2001; 47(2): 85-109.

19. Hawk C, Hyland JK, Rupert R, Colonvega M, Hall S. Assessment of balance and risk for falls in a sample of community-dwelling adults aged 65 and older. Chiropr Osteopat 2006; 14(3): 1-8.

20. Guimarães JMN, Faninatti PTV. Análise descritiva de variáveis teoricamente associadas ao risco de quedas em mulheres idosas . Revista brasileira de medicina do esporte 2005; 11(5): 298-305.

21. Tinetti ME. Factors associated wich serious injury during falls by ambulatory nursing home residents. J Am Geriatr Soc 1987; 35: 644-48.

22. Santariano W A, Haight TJ, Tager IB Reasons given by older people for limitation or avoidance of leisure time physical activity. J Am Geriatr Soc 2000; 48: 505-12.

23. Koepsell TD. Footwear style and risk of falls in older adults. J Am Geriatr Soc 2004; 52: 1495-501.

24. Perracini MR, Ramos LR. Fatores associados a quedas em uma coorte de idosos residentes na comunidade. Rev Saude Publica 2002; 36(6): 709-16.

25. Rao SS Prevention of falls in older patients. Am Fam Physician 2005; 72 (1): 81-8.

26. Lawlor DA, Patel R, Ebrahim S. Association between falls in elderly women and chronic diseases and drug use: cross sectional study. Prim Care 2003; 327(27): 1-6.

27. American Geriatrics Society. British Geriatrics Society. American Academy Orthopaedic Surgeons. Panel on Falls Prevention: guideline for the prevention of falls in older persons. J Am Genatr Soc 2001; 49: 664-72. 